\title{
Heterocromia de íris: uma revisão das condições que podem afetar a pigmentação iridiana
}

\author{
Heterochromia: a review of conditions that may affect iris pigmentation

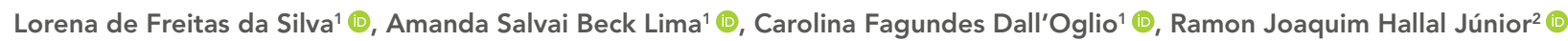

Descritores:

Heterocromia; Íris; Doenças da íris; Pigmentação; Oftalmologia

Keywords: Heterochromia; Iris; Iris diseases; Pigmentation; Ophthalmology.

Recebido: 9/2/2021

Aceito: 4/7/2021

Autor correspondente: Lorena de Freitas da Silva Avenida das Torres, 500 CEP: 85806-095 - Cascavel, PR, Brasil E-mail: lorenadefreitasdasilva@hotmail.com

Instituição de realização do trabalho: Centro Universitário Fundação Assis Gurgacz, Cascavel, PR, Brasil.

Fonte de auxílio à pesquisa: trabalho não financiado.

Conflitos de interesse: os autores declaram que não há conflitos de interesses.

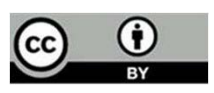

Copyright (C2021

\section{RESUMO}

A íris é responsável pela cor dos olhos. Ela ainda realiza o controle da quantidade de luz que penetra no olho pela pupila. Variações nos genes de cada indivíduo, além da quantidade e da qualidade de melanina na íris, determinam a cor dos olhos. A heterocromia é caracterizada por diferenças na coloração da íris de um mesmo indivíduo, sendo, na maioria das vezes, benigna. Existem basicamente três tipos de heterocromia de íris: central, setorial e completa. A heterocromia de íris pode ter como causa alterações genéticas e congênitas, relacionadas ou não a síndromes específicas, como a de Sturge-Weber, a de Waardenburg, a de Parry-Romberg e a de Horner congênita. Há também causas adquiridas, como doenças ou lesões, trauma ocular e corpos estranhos intraoculares, uso de certas medicações tópicas, siderose ocular, irites ou uveítes como a síndrome uveítica de Fuchs, dentre outras. Diante de um paciente com heterocromia de íris, deve-se entender o contexto e o curso clínico desse sinal, pois pode se tratar de uma alteração de pigmentação benigna ou existir uma doença base em curso, que requer terapêutica específica. Este artigo de revisão de literatura visa abordar as principais etiologias relacionadas à heterocromia de íris, além de discorrer sobre a anatomia e a fisiologia da coloração iridiana e sobre a fisiopatologia de suas possíveis alterações.

\section{ABSTRACT}

The iris is responsible for eye color and controls the amount of light that enters the eye through the pupil. Variation in each individual's genes, besides the quantity and quality of melanin in the iris, determine eye color. Heterochromia is characterized by different colors of irises in the same individual, and it is benign in most cases. There are basically three types of heterochromia: central, partial and complete. Heterochromia can be caused by genetic and congenital alterations, which may or may not be related to specific conditions, such as Sturge-Weber syndrome, Waardenburg syndrome, ParryRomberg syndrome and congenital Horner syndrome. It may be associated to acquired causes like diseases or injuries, such as eye trauma and intraocular foreign bodies, use of some topical medications, ocular siderosis, iritis or uveitis, such as Fuchs' uveitis, among others. When assessing a patient with heterochromia, one must understand the context and clinical course of this signal, since it may be a benign pigmentation disorder or there may be an underlying disease, which requires specific therapy. This literature review article was set out to address the main etiologies related to heterochromia, in addition to describing the anatomy and physiology of the iris color and the pathophysiology of possible alterations. 


\section{INTRODUÇÃO}

A íris é a extensão anterior do cristalino. Ela possui uma abertura central redonda, a pupila, e divide o espaço entre a córnea e o cristalino em câmara anterior e câmara posterior. Sua função é controlar a quantidade de luz que entra pela pupila. É composta de duas camadas de tecidos embriológicos diferentes, sendo que a camada anterior, ou estroma da íris, é derivada do mesoderma e a posterior, do neuroectoderma. ${ }^{(1)}$ As camadas da íris são a borda anterior, o estroma, as fibras musculares e o epitélio pigmentado. ${ }^{(2)}$

A cor da íris é determinada principalmente pela distribuição e pela concentração dos melanócitos, ${ }^{\left({ }^{3}\right)}$ células especializadas na produção de melanina. Os melanócitos formam, posteriormente, o epitélio pigmentado da íris, ${ }^{(4)}$ o qual consiste em duas camadas de células cuboides, ${ }^{(5)}$ e, anteriormente, formam a borda do estroma da íris. ${ }^{(4)}$ Acredita-se que a propriedade de absorção dos componentes da matriz extracelular da íris também influencia em sua coloração, ${ }^{(1,4)}$ assim como a natureza do pigmento de melanina nos melanócitos. ${ }^{(1)}$ Além disso, outro componente que interfere nesse contexto é a córnea, com aspectos como sua opacidade, espessura ou curvatura afetando a cor da íris e o modo como a luz é refletida nela. ${ }^{(6)}$

Existem dois tipos principais de melanina na íris: a eumelanina e a feomelanina. ${ }^{(5)}$ Os olhos de cor mais clara possuem maior quantidade de feomelanina, ${ }^{(2)}$ um pigmento que varia do amarelo ao vermelho, ${ }^{(5)}$ enquanto nos olhos mais escuros, há maior predomínio da eumelanina, ${ }^{(2)}$ apresentando coloração que varia do preto ao marrom. ${ }^{(5)}$ Atualmente, há evidências de que as diferentes cores da íris ocorrem, sobretudo, devido a esses pigmentos contidos nos melanócitos estromais, já que o número de melanócitos parece ser constante nas diferentes cores. ${ }^{(5)}$

A cor da íris é totalmente desenvolvida na infância e não sofre grandes modificações ao longo da vida. ${ }^{(4)}$ Porém, a cor da íris adulta é diferente da cor da íris do recém-nascido. Muitas crianças caucasianas nascem com os olhos azuis e, ao longo dos anos, essa pigmentação pode aumentar, principalmente durante o primeiro ano de vida, embora esse processo possa durar até 6 anos ou mais. ${ }^{(6)}$

A coloração do olho é determinada por vários genes, como HERC2/OCA2, TYRP1, MAPT e MYO5A. ${ }^{(1)}$ Uma troca de base no íntron 86 no lócus do gene HERC2 explica quase todas as associações dos olhos azul-marrom. ${ }^{(6)}$ Além disso, a mutação do gene OCA2 é uma das causas do albinismo óculo-cutâneo tipo 2. ${ }^{(1)}$ A maior parte das variações de cores do olho humano é explicada por três nucleotídeos polimórficos localizados no íntron 1 do gente OCA2. ${ }^{(7)}$
A heterocromia de íris é caracterizada por diferenças de coloração da íris, as quais podem ser hipercrômicas ou hipocrômicas. É, em geral, rara, sendo que a maioria dos casos é benigna, esporádica e ocorre sem outra anormalidade detectável. Apesar de incomum em humanos, é comum em algumas raças de cachorros, gatos e cavalos. ${ }^{\left({ }^{(8)}\right.}$

A alteração de cor pode ocorrer somente em um olho ou em ambos ${ }^{(9)}$ e ser parcial, central ou completa. Na heterocromia completa, ou heterocromia iridis, cada olho possui sua íris com uma coloração específica. Esse tipo de heterocromia é mais rara, afetando menos de 200 mil pessoas nos Estados Unidos. ${ }^{(8)}$ A heterocromia central ocorre quando existe um anel interno que irradia da pupila em direção ao meio da íris, com uma cor diferente da área externa da íris. Na heterocromia parcial, ou heterocromia iridum, apenas uma porção da íris possui uma coloração distinta de seu restante (Figura 1). ${ }^{(10)}$

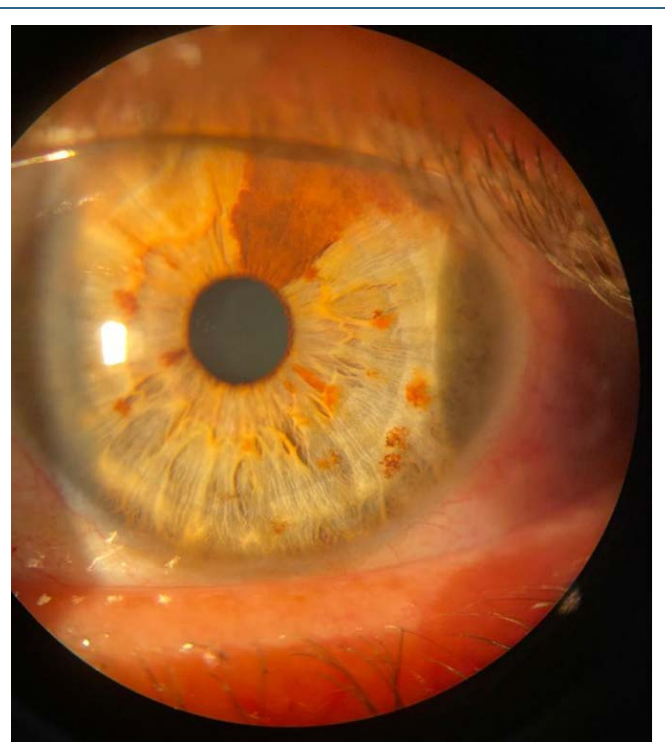

Figura 1. Heterocromia parcial.

Levando em consideração que a heterocromia de íris é uma condição incomum na espécie humana, mas que envolve diversas causas e patologias, faz-se necessária uma abordagem mais aprofundada a respeito do tema. Trata-se de um tópico bastante relevante e que, muitas vezes, não é abordado durante a graduação.

Este artigo pretende contribuir com o conhecimento de acadêmicos e profissionais de saúde, além de complementar as publicações já existentes sobre o assunto. Os autores objetivam expor, de maneira clara, as causas da heterocromia iridiana, que incluem a síndrome uveítica de Fuchs (SUF), a síndrome de Horner (SH), o uso tópico de análogo de prostaglandinas, o nevo de Ota, a siderose 
ocular, o melanoma da íris, a síndrome de Sturge-Weber (SSW) e a síndrome de Waardenburg (SW), dentre outras. Pretende-se explanar sobre essa condição oftalmológica para médicos e acadêmicos, para que o diagnóstico e, se necessário, a investigação e o tratamento desses pacientes sejam realizados da forma mais adequada e prudente possível.

\section{ANÁLISE DAS CAUSAS DE HETEROCRO- MIA DE ÍRIS}

A heterocromia de íris pode ser congênita, relacionada ou não a uma síndrome, ou adquirida por uma doença ou lesão.(9)

\section{CONDIÇÕES CONGÊNITAS}

As principais causas congênitas incluem a heterocromia benigna e as síndromes de Sturge-Weber, de Waardenburg, ${ }^{(8,11)}$ de Parry-Romberg ${ }^{(8)}$ e de Horner congênita. ${ }^{(8,11,12)}$ Existe um caso de heterocromia iridum por herança autossômica dominante envolvendo três gerações de uma família relatado na literatura, que se apresentou em ambos os olhos. A heterocromia iridum pode também ser causada por mosaicismo genético e quimerismo. ${ }^{(13)}$

\section{Síndrome de Sturge-Weber}

A SSW, ou angiomatose encefalotrigeminal, é uma rara desordem congênita ${ }^{(14)}$ e esporádica, ${ }^{(11)}$ apesar de haver, na literatura, descrição de casos herdados de forma autossômica dominante e recessiva, ${ }^{(14)}$ que compõem um conjunto de afecções classificadas como facomatoses ou hamartomatoses, ${ }^{(15)}$ as quais se configuram pela existência de tumores congênitos advindos de tecido usualmente situado na região acometida. ${ }^{(16)}$ Recentemente, foi encontrada uma associação da SSW com uma mutação no gene GNAQ. ${ }^{(17)}$

Trata-se de uma síndrome neuro-oculocutânea, ${ }^{(16)}$ sem predileção sexual ou racial, ${ }^{(15)}$ cuja tríade clássica é a malformação capilar dérmica na distribuição de um ou mais ramos do nervo trigêmeo, particularmente seguindo a trajetória do primeiro e do segundo ramos (nervos oftálmico e maxilar, respectivamente), comumente conhecida como nevus flameus, ou mancha em vinho do Porto, além de angiomatose leptomeníngea ipsilateral à lesão cutânea e envolvimento ocular. ${ }^{(11,18,19)}$ Manifestações orais variadas também podem ocorrer. ${ }^{(20)}$ Apesar de não ser um achado essencial da síndrome, ${ }^{(14)}$ a mancha em vinho do Porto é sua manifestação clínica mais comum, ${ }^{(20)}$ aparecendo na maioria dos casos de SSW. ${ }^{(16)}$ Geralmente, ela está presente ao nascimento e pode progredir ao longo do tempo. ${ }^{(16)} \mathrm{O}$ hemangioma ipsilateral das leptomeninges também é um dos principais sinais da síndrome e, assim como as alterações dermatológicas, pode apresentar caráter progressivo. ${ }^{(14)}$

O envolvimento ocular da SSW, normalmente ipsilateral ao angioma cutâneo, ${ }^{(21)}$ está presente em cerca de $50 \%$ a $60 \%$ dos pacientes ${ }^{(18,21)}$ e se caracteriza por malformações vasculares, que envolvem a conjuntiva, a episclera, a coroide e a retina, ${ }^{(20,21)}$ além do glaucoma. ${ }^{(14)} \mathrm{O}$ glaucoma é o achado ocular mais frequente, podendo afetar de $30 \%$ a $70 \%$ dos pacientes, ${ }^{(18,22)}$ e pode estar presente ao nascimento ou se desenvolver mais tarde. ${ }^{(23)}$ É causado por malformações no ângulo da câmara anterior, o que gera obstrução ao escoamento do humor aquoso, e, ainda, aumento de pressão nas veias episclerais, devido à existência de shunts arteriovenosos no hemangioma, promovendo alterações na hemodinâmica ocular por desordem no fluxo do humor aquoso. ${ }^{(16,17)}$. Em um estudo com 55 pacientes com SSW, 55\% apresentaram hemangioma em coroide e $69 \%$ conjuntival ou escleral. ${ }^{(16)}$ Os hemangiomas coroidais podem ser clinicamente divididos em formas localizadas e difusas, mas é a forma difusa que geralmente ocorre em pacientes com SSW. ${ }^{(21)}$ A coroide pode se tornar significativamente mais espessa, ${ }^{(16)}$ e tem sido relatado um risco aumentado de desenvolvimento de glaucoma e complicações retinianas graves. ${ }^{(21)}$

Outras anormalidades oculares menos frequentes, como coloboma da íris, ${ }^{(14)}$ catarata $^{(21)}$ e outras deformidades do cristalino, ${ }^{(14)}$ estrabismo, descolamento de retina, ${ }^{(16)}$ além da heterocromia de íris, ${ }^{(16,17,21,22)}$, já foram relatadas. A heterocromia de íris é, assim, um achado menos comum na SSW, porém pode estar presente.

\section{Síndrome de Waardenburg}

A SW consiste em um grupo de doenças genéticas que possuem um quadro clínico de perda auditiva e alterações pigmentares de olhos, cabelo e pele. É uma condição rara ${ }^{(24,25)}$ do desenvolvimento da crista neural e possui prevalência de 1:42 mil até 1:50 mil. ${ }^{(25)}$ Na maioria dos casos, a síndrome possui um padrão autossômico dominante, ${ }^{(25,26)}$ mas, no subtipo IV da doença, pode haver um padrão autossômico recessivo. ${ }^{(1)}$ A SW possui quatro subtipos principais, de acordo com as manifestações clínicas.

Em relação ao contexto genético da doença, a SW tipo I e a tipo III são causadas por mutações no gene PAX $3{ }^{(1,27)}$ enquanto a SW tipo II é causada por uma mutação do gene do fator de transcrição associado à microftalmia, ${ }^{(1,27)}$ e a SW tipo IV está associada a mutações do fator de transcrição SOX-10, do receptor de endotelina tipo B e da endotelina 3. ${ }^{(27)}$ 
Os subtipos mais comuns são o I e o II, e o maior diferencial entre eles é a presença da distopia canthorum (prega cutânea que se prolonga da base do nariz até o final da região medial da sobrancelha), (24) a qual ocorre com maior frequência na SW tipo I. ${ }^{(27)}$ Em ambos os tipos, há perda auditiva e distúrbios de pigmentação, ${ }^{(25)}$ porém a perda auditiva e a heterocromia de íris são mais comuns na SW tipo II. ${ }^{(28)}$

A SW tipo III, também chamada de síndrome de Klein-Waardenburg, além de apresentar as manifestações presentes na SW tipo I, também tem anormalidades dos membros superiores. ${ }^{(27,29)}$ Ademais, pode estar associada ao fechamento incompleto do céu da boca e/ou fenda labial no lábio superior. ${ }^{(29)}$

A SW tipo IV, ou síndrome de Waardenburg-Shah, é muito rara ${ }^{(25,29,30)}$ e caracterizada por distúrbios pigmentares, surdez e doença de Hirschsprung (anomalia congênita da inervação do intestino baixo, que resulta em obstrução parcial ou total do intestino). ${ }^{(31)}$ Pode haver também heterocromia de íris e anormalidades da cor do cabelo. ${ }^{\left({ }^{22}\right)}$ Além disso, um dos primeiros sintomas que pode aparecer nessa doença é uma descoloração azulada dos olhos. ${ }^{(33)}$

Na SW, podem ser encontradas a heterocromia parcial, total ou segmentar da íris, que pode ser tanto unilateral como bilateral. ${ }^{(1)}$

\section{CONDIÇÕES ADQUIRIDAS}

Dentre as principais causas adquiridas de heterocromia iridiana, citam-se o corpo estranho intraocular, ${ }^{(34)}$ o trauma ocular, $^{(8,9)}$ a SH adquirida tardiamente, ${ }^{(8,10,35)}$ a oclusão da veia central da retina, a siderose, ${ }^{(11)}$ o diabetes mellitus, os tumores benignos na íris, cistos ou abscessos, o melanoma ou nevo difuso da íris, ${ }^{(8,11,36)}$ as irites ou uveítes, o uso unilateral tópico de um análogo da prostaglandina para o glaucoma, ${ }^{(8,1,1,35)}$ a $S \mathrm{SF}^{(9,11)}$ e a quimioterapia intravítrea. ${ }^{(37)}$ Diversos agentes etiológicos que levam à uveíte podem promover uma irite heterocrômica, como a uveíte herpética, ${ }^{(9,35)}$ incluindo o citomegalovírus associado à síndrome de Fuchs, ${ }^{(38)}$ além do Ebolavirus ${ }^{(39)}$ e do Rubella vírus associado à síndrome de Fuchs. ${ }^{\left({ }^{38}\right)}$ A melanocitose oculodermal (nevo de Ota) também é uma causa relatada de heterocromia de íris. ${ }^{(11,40)}$ Áreas de hipopigmentação da íris comumente vistas em idosos podem ocorrer devido à atrofia senil da íris. ${ }^{\left({ }^{\circ o)}\right.}$ Há um caso de trauma ocular ao nascimento causado por fórceps que se apresentou como heterocromia isolada. ${ }^{(41)}$

\section{Síndrome uveítica de Fuchs}

A SUF, ou iridociclite heterocrômica de Fuchs, é uma condição ocular crônica, caracterizada por uma uveíte tipicamente não granulomatosa e unilateral em 90\% dos casos. ${ }^{(11,42)}$ Ocorre em aproximadamente $2 \%$ a 11,5\% dos casos de uveíte, ${ }^{(43)}$ principalmente na terceira e quarta décadas de vida, e acomete igualmente ambos os sexos. ${ }^{(42)}$ A incidência da SUF é estimada entre 1,8\% e 22,7\%, em países desenvolvidos, e entre zero e 5,6\%, nos países em desenvolvimento. ${ }^{(44)}$

Trata-se de uma afecção de início insidioso e baixo grau de atividade, apesar de possuir um espectro amplo de apresentações clínicas. ${ }^{(44)}$ O portador de SUF pode ser inicialmente assintomático por anos. ${ }^{(42)}$ Geralmente é caracterizada por precipitados queráticos difusos estrelados, atrofia da íris com ou sem heterocromia, ausência de sinéquias posteriores, opacidades vítreas, reação celular anterior de baixo grau e inexistência de sintomas agudos, como fotofobia, olho vermelho ou mesmo dor. ${ }^{(43,44)}$ Recentemente, um afinamento da coroide tem sido encontrado em pacientes com SUF. ${ }^{(44)}$ A maioria dos pacientes apresenta alterações visuais especialmente devido a complicações secundárias, como catarata e glaucoma. ${ }^{(45)}$ A catarata é a complicação mais frequente da síndrome, ${ }^{(42)}$ possuindo prevalência de $23 \%$ a 90,7\%. Já o glaucoma secundário está presente em 15\% a 59\% dos pacientes. ${ }^{(45)}$

A heterocromia não hereditária da íris é um sinal comum e importante, ${ }^{(11)}$ sendo encontrada em cerca de 13,9\% dos casos. ${ }^{(45)}$ Ela pode preceder alguma manifestação clínica visual, e o diagnóstico da síndrome pode ser realizado na presença ou na ausência desse sinal. ${ }^{(42,43)} \mathrm{O}$ aspecto da heterocromia é definido por relativos níveis de atrofia estromal e do pigmento do epitélio posterior e, também, pela coloração iridiana natural do paciente. ${ }^{(11)} \mathrm{A}$ heterocromia é observada predominantemente em pacientes com íris de cor mais clara. Pacientes com íris de cor mais escura têm mais células pigmentares na camada da borda anterior da íris, o que torna a atrofia na íris menos aparente e, desse modo, desenvolverão menos comumente uma heterocromia evidente. ${ }^{(45)}$ É mais habitual que o olho acometido seja hipocrômico, porém, em olhos azuis, o estroma atrofiado pode permitir que a camada pigmentada posterior se realce, tornando-se a pigmentação principal, e, dessa forma, poderá ocorrer uma hipercromia nesse olho. ${ }^{(11)}$

\section{Uso tópico de análogo das prostaglandinas}

Análogos de prostaglandina são comumente utilizados para o tratamento do glaucoma, devido ao seu potencial de reduzir a pressão ocular, drenando-se o humor aquoso pela via úveo-escleral. A pigmentação da íris pode ser induzida por uso de análogos da prostaglandina para o tratamento de glaucoma. ${ }^{(46)}$ Os principais utilizados são latanoprosta (análogo da prostaglandina F2 $\alpha$ ), isopropil, 
unoprostona, travoprosta e bimatoprosta. Estudos demonstraram que o uso de latanoprosta possui maior relação com a heterocromia de íris quando comparado com o uso dos outros medicamentos citados. ${ }^{(8,46)}$ Dessa forma, a aplicação da droga latanoprosta pode aumentar a pigmentação da íris em alguns pacientes, mas a fisiopatologia do mecanismo ainda requer mais estudos. ${ }^{(5)}$ Além do uso dessas drogas para o tratamento do glaucoma, os análogos de prostaglandina, especialmente o bimatoprosta (nome comercial Latisse ${ }^{\circledR}$ ), estão sendo popularmente comercializados para alongamento dos cílios. O tratamento tópico da droga Latisse ${ }^{\circledR}$ nos cílios aumenta a taxa de porcentagem dos folículos ciliares na fase anágena e estimula a melanogênese, resultando em cílios mais longos e escuros. Ademais, pode aumentar o tamanho da papila dérmica e do bulbo papilar, deixando os cílios com a espessura mais grossa. ${ }^{(47)}$ Assim, o uso de prostaglandinas com o objetivo de alongar os cílios pode induzir a distúrbios estéticos, como a heterocromia de íris, e também a distúrbios funcionais da pálpebra, causando a migração posterior dos cílios, podendo resultar em blefarofimose adquirida. ${ }^{(48)}$

\section{Melanocitose oculodermal (nevo de Ota)}

O nevo de Ota, um tipo de melanocitose oculodermal, é uma anormalidade pigmentar congênita ${ }^{(49,50)}$ rara, ${ }^{(51)}$ que se manifesta com uma mancha hiperpigmentada acinzentada ou preto azulada e afeta principalmente os ramos oftálmico e maxilar do nervo trigêmeo. ${ }^{\left({ }^{2} 2\right)}$ Essa hiperpigmentação pode atingir a pele periorbital, a esclera, a úvea, a órbita, as meninges, o palato e a membrana timpânica ${ }^{(50)}$, sendo que o acometimento oral é raro. ${ }^{(51)}$ Além disso, o paciente geralmente possui heterocromia de íris, que adquire uma cor mais escurecida. ${ }^{(49)}$

Na maioria dos casos, o nevo de Ota ocorre de forma unilateral| ${ }^{(49,50)}$ (em cerca de $\left.95 \%\right)^{(52)}$ e é mais prevalente em asiáticos e afrodescendentes. ${ }^{(49)}$ Geralmente, a criança já nasce com essa anormalidade, ${ }^{(52)}$ mas a mancha pode escurecer em períodos de alta taxa metabólica, como na puberdade e na gravidez. ${ }^{(49)}$

Apesar de essa condição normalmente ser benigna, ${ }^{(52)}$ o aumento da densidade dos melanócitos da íris, do corpo ciliar e da coroide nesses pacientes representa um maior risco para desenvolvimento de melanoma uveal ao longo da vida. ${ }^{(53)}$ Estima-se que o risco de um paciente caucasiano com melanocitose oculodermal desenvolver melanoma uveal é de um em $400^{(50)}$. Além disso, esses pacientes são mais propensos ao desenvolvimento de glaucoma, devido ao acúmulo de melanócitos. ${ }^{(49)}$

\section{Siderose ocular}

A siderose ocular é causada por lesões intraoculares de corpos estranhos que contenham partículas de ferro e caracteriza-se por alterações pigmentares e degenerativas. Essa doença afeta principalmente jovens que trabalham em indústrias com manuseio de materiais de ferro, nas quais podem ocorrer traumas oculares em que fragmentos de metais penetram o olho com grande afinidade por superfícies epiteliais, afetando principalmente íris, epitélio não pigmentado ciliar, lente e epitélio pigmentar da retina. ${ }^{(54,55)}$ A siderose ocorre por depósito de agregados de partículas de ferritina aderidas em corpos densos ligados à membrana intracitoplasmática (siderossomos) nas células epiteliais do olho. ${ }^{(56,57)} \mathrm{A}$ ferritina cursa com degeneração vacuolar, e os depósitos de ferro alteram a cor da íris, resultando na heterocromia. ${ }^{(58)}$

As consequências da siderose, dessa forma, incluem heterocromia de íris, midríase pupilar, depósitos marrons na cápsula anterior da lente, formação de catarata e degeneração do pigmento da retina. ${ }^{(55)}$ Um estudo analisando 24 pacientes com siderose bulbosa submetidos à cirurgia demonstrou que as complicações mais frequentes dessa afeç̧ão foram catarata (95,83\%), degeneração pigmentar da retina $(68,18 \%)$, heterocromia da íris (58,33\%), midríase pupilar $(47,62 \%)$, glaucoma secundário $(25 \%)$, defeito pupilar aferente relativo (25\%) e descolamento de retina $(12,50 \%)$. A heterocromia de íris, portanto, é um dos achados na siderose ocular, mas os pacientes podem não notar a alteração de cor. ${ }^{(55)}$

\section{Melanoma da íris}

O melanoma é a malignidade primária mais comum da íris, mas é responsável por apenas 3\% a 10\% de todos os melanomas uveais. ${ }^{(59,60)}$ A idade média dos pacientes com diagnóstico de melanoma de íris é de meados ao final dos 40 anos de idade, sem predileção por sexo ou lateralidade do olho. ${ }^{(61)}$

Além da pele de cor clara e da coloração da íris de azul a cinza, existem poucos fatores de risco conhecidos para o melanoma da íris. ${ }^{(60,61)}$ Quanto à associação do melanoma uveal à exposição solar, estudos epidemiológicos produziram resultados inconsistentes. ${ }^{(61)}$

Os melanomas de íris exibem dois padrões de crescimento: circunscrito e difuso. Melanomas de íris circunscritos comumente se apresentam como uma mancha pigmentada assintomática. ${ }^{(61)}$ Já o melanoma difuso de íris, por outro lado, é uma variante rara. É responsável por aproximadamente $10 \%$ dos melanomas da íris. ${ }^{(62,63)}$ Geralmente cursa com íris escura unilateral (heterocromia 
hipercrômica progressiva) sem espessamento focal e glaucoma unilateral secundário, que normalmente é devido ao envolvimento do tumor na rede trabecular. ${ }^{(61,62)}$

\section{Síndrome de Horner}

A SH é o resultado do bloqueio da via óculo-simpática, que pode acometer desde o hipotálamo até a órbita. ${ }^{(64,65)}$ Os sintomas dependem se a lesão for pré-ganglionar ou pós-ganglionar. ${ }^{(66)}$ A tríade clássica da síndrome compreende ptose ipsilateral, miose e anidrose facial. ${ }^{(66,67)} \mathrm{A}$ SH afeta aproximadamente uma a cada 6 mil pessoas, sem predileção de faixas etárias e etnias. ${ }^{(68)}$ A etiologia pode ser adquirida, congênita ou hereditária. ${ }^{(68)} \mathrm{A}$ condição adquirida é secundária a doenças sistêmicas, locais ou de causas iatrogênicas, que cursam com danos no suprimento simpático. Já a SH congênita e a hereditária são mais raras. ${ }^{(68)}$

Além da tríade clássica, essa síndrome pode ter associação com heterocromia da íris, chamada de síndrome de Von Passow. Essa heterocromia pode ser observada em crianças menores de 2 anos de idade e na forma congênita, sendo incomum na SH adquirida. ${ }^{(66,68,69)}$ A heterocromia na SH ocorre devido à redução do número de melanócitos da íris, mecanismo semelhante às modificações fisiológicas da cor dos olhos na idade adulta. A heterocromia é decorrente também da estimulação simpática que cursa com efeito trófico nos melanócitos da íris, cuja desnervação reduz o número de melanócitos, tanto na borda anterior quanto nas camadas estromais iridianas, tornando o olho afetado mais claro. ${ }^{(1,69)}$

\section{CONDIÇÕES HIPERCRÔMICAS E HIPOCRÔMICAS}

Alguns autores sugerem também a divisão das causas de heterocromia de íris em condições que resultam em diminuição da pigmentação da íris ou de uma porção desta e condições que aumentam a pigmentação. Dentre as que resultam em hipocromia iridiana, podem-se mencionar: SW, SH, síndrome de Parry-Romberg, irites crônicas, SUF, carcinomas metastáticos, xantugranuloma juvenil, dentre outras. ${ }^{(5)}$ Já o diagnóstico diferencial de uma íris hipercrômica inclui nevo difuso da íris ou melanoma, melanose ocular, hemossiderose ocular, siderose ocular de um corpo estranho de ferro retido, atrofia essencial da íris, glaucoma melanocitomalítico e aumento da melanogênese por agonistas de prostaglandina tópicos. ${ }^{(5,61)}$

A principal limitação deste estudo consistiu na carência de publicações científicas que abordassem a heterocromia como um sinal clínico secundário a uma patologia e discutissem os diagnósticos diferenciais.

\section{CONSIDERAÇÕES FINAIS}

Diante de um paciente com heterocromia, é de extrema importância o entendimento do contexto e do curso clínico desse sinal, pois pode se tratar de uma alteração congênita, adquirida ou derivada de alguma patologia. A análise de outras manifestações e da história clínica do paciente em questão possibilita essa diferenciação. Pacientes com heterocromia iridis devem ser submetidos a uma investigação oftalmológica adequada, pois pode significar uma doença de base em curso e necessitar de um tratamento específico.

\section{REFERÊNCIAS}

1. Rennie IG. Don't it make my blue eyes brown: heterochromia and other abnormalities of the iris. Eye (Lond). 2012;26(1):29-50.

2. Sturm RA, Larsson M. Genetics of human iris colour and patterns. Pigment cell \& melanoma research. 2009;22(5):544-62.

3. Tomar M, Dhiman R, Sharma G, Yadav N. Artistic Iris: A Case of Congenital Sectoral Heterochromia Iridis. J Ophthalmic Vis Res. 2018;13(3):359-60.

4. Sun HP, Lin Y, Pan CW. Iris color and associated pathological ocular complications: a review of epidemiologic studies. Int J Ophthalmol. 2014;7(5):872-8

5. Imesch PD, Wallow IH, Albert DM. The color of the human eye: a review of morphologic correlates and of some conditions that affect iridial pigmentation. Surv Ophthalmol. 1997;41 Suppl 2:S117-23.

6. Mackey DA, Wilkinson $\mathrm{CH}$, Kearns LS, Hewitt AW. Classification of iris colour: review and refinement of a classification schema. Clin Exp Ophthalmol. 2011;39(5):462-71.

7. Duffy DL, Montgomery GW, Chen W, Zhao ZZ, Le L, James MR, et al. A threesingle-nucleotide polymorphism haplotype in intron 1 of OCA2 explains most human eye-color variation. Am J Hum Genet. 2007;80(2):241-52.

8. Ur Rehman H. Heterochromia. CMAJ. 2008;179(5):447-8.

9. Mackey DA, Wilkinson $\mathrm{CH}$, Kearns LS, Hewitt AW. Classification of iris colour: review and refinement of a classification schema. Clin Exp Ophthalmol. 2011;39:462-71

10. Gladstone RM. Development and significance of heterochromia of the iris. Arch Neurol. 1969;21(2):184-91.

11. Kanski JJ, Bowling B, Nischal K, Pearson A. Oftalmologia clínica: uma abordagem sistêmica. Amsterdan: Elsevier; 2012.

12. Olivera RB, Fernández L, Arias AP, Ballarino D, Jara A. Síndrome de Horner congénito. Caso clínico. Arch Argent Pediatr. 2018;116:85-7.

13. Tomar M, Dhiman R, Sharma G, Yadav N. Artistic iris: A case of congenital sectoral heterochromia iridis. J Ophthalmic Vis Res. 2018;13(3):359-60.

14. Palheta Neto FX, Vieira Júnior MA, Ximenes LS, Jacob CC, Rodrigues Júnior AG, Palheta AC. Aspectos Clínicos da Síndrome de Sturge-Weber. Arq Int Otorrinolaringol. 2008;12(4):565-70.

15. Wong HS, Abdul Rahman R, Choo SY, Yahya N. Sturge-Weber-Syndrome with extreme ocular manifestation and rare association of upper airway angioma with anticipated difficult airway. Med J Malaysia. 2012;67(4):435-7.

16. Figueiredo LR, Silva Filho FJ, Rehder JR. Síndrome de Sturge-Weber e suas repercussões oculares: revisão da literatura. Rev Bras Oftalmol. 2011;70:194-9.

17. Velásquez-Gallego C, Ceballos-Ruiz JF, Ruiz-Jaramillo N, VillamizarLondoño C. Síndrome de Sturge-Weber: Reporte de un caso y revisión de la literatura. Revt Ecuatoriana Neurol. 2019;28(2):105-14.

18. Café ME, Rodrigues RC, Viggiano AM. Você conhece esta síndrome? An Bras Dermatol. 2008;83:167-9.

19. Plateroti AM, Plateroti R, Mollo R, Librando A, Contestabile MT, Fenicia V. Sturge-Weber Syndrome Associated with Monolateral Ocular Melanocytosis, Iris Mammillations, and Diffuse Choroidal Haemangioma. Case Rep Ophthalmol. 2017;8(2):375-84. 
20. Hassan S, Babiker A, Bashiri FA, Hassan HH, Husseini ME, Salih MA. Sturge-Weber syndrome: Continued vigilance is needed. Sudan J Paediatr. 2015;15(2):63-70.

21. Mantelli F, Bruscolini A, La Cava M, Abdolrahimzadeh S, Lambiase A. Ocular manifestations of Sturge-Weber syndrome: pathogenesis, diagnosis, and management. Clin Ophthalmol. 2016;10:871-8.

22. Chhabria BA, Subramanium PB, Nampoothiri R, Bhalla A, Varma S. SturgeWeber Syndrome. J Clin Diagn Res. 2017;11(2):OJ05-OJ6.

23. Higueros E, Roe E, Granell E, Baselga E. Sturge-Weber Syndrome: A Review. Actas Dermosifiliogr. 2017;108(5):407-17.

24. Bocángel MA, Melo US, Alves LU, Pardono E, Lourenço NC, Marcolino HV, et al. Waardenburg syndrome: Novel mutations in a large Brazilian sample. Eur J Med Genet. 2018;61(6):348-54.

25. Rawlani SM, Ramtake R, Dhabarde A, Rawlani SS. Waardenburg syndrome: A rare case. Oman J Ophthalmol. 2018;11(2):158-60.

26. Shelby MV. Waardenburg syndrome expression and penetrance. J Rare Dis Res Treat. 2017;2(6):31-40.

27. Dave TV, Pappuru RR, Dave VP. When color matters: Waardenburg syndrome. Indian J Ophthalmol. 2019;67(1):135

28. Milunsky JM. Waardenburg Syndrome Type I. In: Adam MP, Ardinger HH, Pagon RA, Wallace SE, Bean LJ, Stephens K, et al. (eds.). GeneReviews(®). Seattle (WA): University of Washington, Seattle

29. Shrinkhal, Singh A, Mittal SK, Agrawal A, Verma R, Yadav P. Waardenburg syndrome with dry eyes: A rare association. Taiwan J Ophthalmol. 2019;9(3):198-201.

30. Mahmoudi A, Rami M, Khattala K, Elmadi A, Afifi MA, Youssef B. ShahWaardenburg syndrome. Pan Afr Med J. 2013;14:60.

31. Hogan AR, Rao KA, Thorson WL, Neville HL, Sola JE, Perez EA. Waardenburg syndrome type iv de novo SOX10 variant causing chronic intestinal pseudoobstruction. Pediatr Gastroenterol Hepatol Nutr. 2019;22(5):487-92.

32. Chandra Mohan SL. Case of Waardenburg Shah syndrome in a family with review of literature. J Otol. 2018;13(3):105-10.

33. Pattebahadur R, Singhi S, Maharana PK. Waardenburg-Shah syndrome: a rare case in an Indian child. BMJ Case Rep. 2016;2016.

34. Barr CC, Vine AK, Martonyi CL. Unexplained heterochromia. Intraocular foreign body demonstrated by computed tomography. Surv Ophthalmol. 1984;28(5):409-11.

35. Beynat J, Soichot P, Bidot S, Dugas B, Creuzot-Garcher C, Bron A. Hétérochromie irienne associée à un syndrome de Claude Bernard-Horner acquis. J Fr Ophtalmol. 2007;30.

36. Demirci H, Shields CL, Shields JA, Eagle RC Jr, Honavar SG. Diffuse iris melanoma: a report of 25 cases. Ophthalmology. 2002;109(8):1553-60.

37. Camp DA, Lally SE, Shields CL. Heterochromia following intravitreal chemotherapy in two cases. J AAPOS. 2019;23(4):241-3.

38. Chan NS, Chee SP. Demystifying viral anterior uveitis: a review. Clin Exp Ophthalmol. 2019;47(3):320-33.

39. Shantha JG, Crozier I, Varkey JB, Kraft CS, Lyon GM 3rd, Mehta AK, et al. Long-term Management of Panuveitis and Iris Heterochromia in an Ebola Survivor. Ophthalmology. 2016;123(12):2626-8.e2.

40. Cronemberger S, Calixto N, Freitas HL. Nevus of Ota: clinicalophthalmological findings. Rev Bras Oftalmol. 2011;70(5):278-83.

41. Simpson SM, Yau G, Nischal KK, Strube YN. Forceps trauma in a newborn presenting as iris heterochromia. J AAPOS. 2017;21(5):425-6.

42. Cordeiro MC, Barros RA, Rehder JR. Glaucoma secundário à iridociclite heterocrômica de Fuchs. Rev Bras Oftalmol. 2011;70(2):114-7.

43. Guedes M, Garcia F. Uveíte Heterocrômica de Fuchs. Gazeta Médica. 2016;3(2):59-60

44. Sun Y, Ji Y. A literature review on Fuchs uveitis syndrome: an update. Surv Ophthalmol. 2020;65(2):133-43
45. Ronquillo MMAVY. Fuchs Uveitis Syndrome. StatPearls Publishing, Treasure Island (FL). 2020.

46. Stjernschantz JW, Albert DM, Hu DN, Drago F, Wistrand PJ. Mechanism and clinical significance of prostaglandin-induced iris pigmentation. Surv Ophthalmol. 2002;47 Suppl 1:S162-75.

47. Cohen JL. Enhancing the growth of natural eyelashes: the mechanism of bimatoprost-induced eyelash growth. Dermatol Surg. 2010;36(9):136171.

48. Custer PL, Kent TL. Observations on prostaglandin orbitopathy. Ophthalmic Plast Reconstr Surg. 2016;32(2):102-5.

49. Swann PG. The fundus in oculodermal melanocytosis. Is this a new observation? Clin Exp Optom. 2003;86(3):183-6

50. Kaliki S, Shields CL. Uveal melanoma: relatively rare but deadly cancer. Eye (Lond). 2017;31(2):241-57.

51. Maguire J, Holt D. Nevus of Ota - an intraoral presentation: a case report. J Med Case Rep. 2019;13(1):174.

52. Saha A, Bandyopadhyay D. Nevus of Ota. Indian Pediatr. 2014;51(6):510.

53. Ranjit RU, Leyngold IM, Margo CE. Melanoma-associated spongiform scleropathy in oculodermal melanocytosis with primary orbital melanoma. Ocul Oncol Pathol. 2016;2(4):276-9.

54. Bloom WR, Ramsey JK, Ohr MP. Ocular siderosis secondary to retained intraocular foreign body: a case report. Cureus. 2019;11(5):e4660.

55. Lapira M, Karl D, Murgatroyd H. Siderosis bulbi as a consequence of a missed intraocular foreign body. BMJ Case Rep. 2014;2014.

56. Tawara A. Transformation and cytotoxicity of iron in siderosis bulbi. Invest Ophthalmol Vis Sci. 1986;27(2):226-36.

57. Hope-Ross M, Mahon GJ, Johnston PB. Ocular siderosis. Eye (Lond) 1993;7(Pt 3):419-25.

58. Zhu L, Shen P, Lu H, Du C, Shen J, Gu Y. Ocular trauma score in siderosis bulbi with retained intraocular foreign body. Medicine (Baltimore). 2015;94(39):e1533.

59. Shields CL, Shields JA, Materin M, Gershenbaum E, Singh AD, Smith A. Iris melanoma: risk factors for metastasis in 169 consecutive patients. Ophthalmology. 2001;108:172-8.

60. Shields CL, Materin MA, Shields JA, Gershenbaum E, Singh AD, Smith A. Factors associated with elevated intraocular pressure in eyes with iris melanoma. Br J Ophthalmol. 2001;85(6):666-9.

61. Henderson E, Margo CE. Iris melanoma. Arch Pathol Lab Med. 2008;132:268-72.

62. Demirci H, Shields CL, Shields JA, Eagle RC Jr., Honavar SG. Diffuse iris melanoma: a report of 25 cases. Ophthalmology. 2002;109(8):1553-60.

63. Leblanc A, Lumbroso-Le Rouic L, Desjardins L, Dendale R, Cassoux N Diffuse iris melanoma: conservative treatment with proton beam therapy after limbal stem cell preservation or enucleation? Ocul Oncol Pathol. 2019;5(6):396-401.

64. George ND, Gonzalez G, Hoyt CS. Does Horner's syndrome in infancy require investigation? Br J Ophthalmol. 1998;82(1):51-4

65. Martin TJ. Horner syndrome: a clinical review. ACS Chem Neurosci. 2018;9(2):177-86.

66. Ulusoy MO, Kıvanç SA, Atakan M, Mayalı H. Post-thyroidectomy iatrogenic Horner's syndrome with heterochromia. J Curr Ophthalmol. 2016;28(1):467.

67. Deprez FC, Coulier J, Rommel D, Boschi A. Congenital horner syndrome with heterochromia iridis associated with ipsilateral internal carotid artery hypoplasia. J Clin Neurol. 2015;11(2):192-6.

68. Khan Z, Bollu PC. Horner syndrome. StatPearls. Treasure Island (FL)2020.

69. Beynat J, Soichot P, Bidot S, Dugas B, Creuzot-Garcher C, Bron A. [Iris heterochromia in acquired Horner's syndrome]. J Fr Ophtalmol. 2007;30(7):e19. French. 